\title{
Crystal Structure Reveals a Unique ABIN-Ubs Binding Mode
}

Jhen-Yi Hong ${ }^{a}$, Su-Chang Lin ${ }^{b}$, Yu-Chih Lo

aDepartment of Biotechnology and Bioindusry Sciences, College of Bioscience and Biotechnology, National Cheng Kung University, Tainan 70101, Taiwan, gracelo@mail.ncku.edu.tw

${ }^{\mathrm{b}}$ Genomics Research Center, Academia Sinica, Taipei, Taiwan

ABINs (A20-binding inhibitor of NF-KB activation) is a negative regulator of NF-KB and belongs to UBAN (ubiquitin binding in ABIN and NEMO) family, which can facilitate the interaction with linear poly-ubiquitin chain by the UBAN domain. Here, we report a crystal structure of the complex between ABIN-UBAN domain and linear tetra-ubiquitin. The structure reveals a unique UBAN-Ubs binding mode, in which a parallel coiled-coil dimer of UBAN motif that interacts with tetra-ubiquitin chain in a molecular stoichiometry of 2:1. We further performed the biochemical assays and found that longer ubiquitin chains may cooperate with more ABIN molecules in solution. Collectively, these results may suggest that long polyubiquitin chains can mediate high order aggregations of $A B I N s$. Finally, we identified the important interacting sites and clarify the disease-causing mutations which disrupt the interaction between $A B I N s$ and ubiquitin.

Reference:

[1] SM Lin, SC Lin, JY Hong, TW Su, BJ Kuo, WH Chang, YF Tu, Yu-Chih Lo*. Structural Insights into Linear Tri-Ubiquitin Recognition by A20-Binding Inhibitor of NF-KB (ABIN)-2. Structure. 25, p66-78. (2017) *corresponding author 